\section{A) Check for updates}

Cite this: Mater. Adv., 2021

2, 1083

Received 28th September 2020 ,

Accepted 14th January 2021

DOI: 10.1039/d0ma00742k

rsc.li/materials-advances

\title{
Initial development of preceramic polymer formulations for additive manufacturing
}

\author{
Saja M. Nabat Al-Ajrash, (D) * Charles Browning, Rose Eckerle and Li Cao
}

\begin{abstract}
Three preceramic polymer formulations for potential use in additive manufacturing technologies were investigated. The polymeric precursors include an allyl hydrido polycarbosilane (SMP-10), a mixture of SMP-10 with a reactive ester (1,6-hexanediol diacrylate, HDDA), and a polydimethylsiloxane (4690A/B). The SMP-10/HDDA proved to have outstanding photo-curing properties, high-resolution printing, and the ability to easily transform into the silicon carbide phase. The same polymeric mixture showed the lowest viscosity value which is preferred in vat additive manufacturing. Thermogravimetric analysis showed that, after pyrolysis to $1350{ }^{\circ} \mathrm{C}$, the polydimethylsiloxane polymer showed the highest onset decomposition temperature and the lowest retained weight (52 wt\%) while the allyl hydrido polycarbosilane showed the lowest onset decomposition temperature and highest retained weight (71.7 wt\%). In terms of crystallography, X-ray diffraction and microstructural results showed that the ceramic matrix composites contained both silicon carbide and silicon oxycarbide. Overall, the results are very promising for the fabrication of ceramic materials using additive manufacturing technologies.
\end{abstract}

\section{Introduction}

Common industrial ceramic fabrication processes such as slip and tape casting, ${ }^{1}$ injection molding, ${ }^{2}$ and powder pressing ${ }^{3,4}$ have many limitations. Such processes rely on mold usage which restricts the form of the fabricated ceramic part to relatively simple geometries. To produce a more complex product using these methods, further machining or shaping of the part is necessary. This proves challenging when working with inherently brittle ceramics. In the last few decades, additive manufacturing (AM) technologies have grown in popularity among researchers as they enable the manufacturing of more complex final products. ${ }^{5}$ To successfully print parts with high precision in polymer-based AM, the polymeric precursors must have specific rheological properties. In particular, viscosity is crucial to successful printing. For example, in stereolithography or digital light processing, a polymer with low viscosity, below $5 \mathrm{~Pa} \mathrm{~S}$ is preferred to facilitate layers coating and reduce the printing time. ${ }^{6}$

To produce ceramic parts containing silicon carbide and silicon oxycarbide, the $3 \mathrm{D}$ printed polymeric precursors must have silicon in their main polymeric chain which converts to ceramic material with subsequent pyrolysis in inert gas. ${ }^{7}$ This is a sophisticated process that includes many chemical and physical changes in the polymer to the ceramic route. Adequate knowledge in many fields like inorganic/organic chemistry,

Department of Chemical and Materials Engineering, University of Dayton, 300 College Park, Dayton, Ohio, 45469, USA. E-mail: nabats1@udayton.edu, sajamodher0@gmail.com mineralogy, materials science, and computer-assisted modeling is necessary to study polymer derived ceramics. ${ }^{8}$ Among ceramics fabricated from preceramic polymers, silicon carbide has special importance due to its extraordinary environmental stability, mechanical properties, and resistance to oxidation (until $1400{ }^{\circ} \mathrm{C}$ ) ${ }^{9}$

Eckel et al. ${ }^{10}$ reported a process for the fabrication of a honeycomb structure by using pre-ceramic polymers. The polymers were ultraviolet curable siloxanes prepared by mixing methylsiloxane with vinyl methoxy siloxane. After pyrolyzing at $1000{ }^{\circ} \mathrm{C}$ in inert gas, around $42 \%$ mass loss and $30 \%$ linear shrinkage were reported. The resulting ceramic products showed very limited defects and were fully dense.

Min et al. ${ }^{11}$ fabricated silicon nitride ceramics using digital light processing (DLP) additive manufacturing and subsequent pyrolysis. To optimize the heat treatment of the green body after printing, three distinct pyrolysis temperatures $\left(1200{ }^{\circ} \mathrm{C}\right.$, $1400{ }^{\circ} \mathrm{C}$, and $1600{ }^{\circ} \mathrm{C}$ ) were investigated. The linear shrinkage, ceramic yield, and relative density at each temperature were calculated. It was found that the optimal pyrolysis temperature was near $1400{ }^{\circ} \mathrm{C}$ as it converted the preceramic polymer to a dense ceramic product with improved structural and mechanical properties. Wang et al. ${ }^{12}$ compared the ceramic yield of three different silicon-based polymers after pyrolysis. They applied thiol-ene free-radical addition which works with polymer derived ceramics containing carbon-carbon double bonds. For this study, three distinct polymers were chosen: methylvinylhydrogen polycarbosilazane, liquid methylvinylhydrogenpolysiloxane, and allylhydrydopolycarbosilane. The photoinitiator, phenylbis $(2,4,6-$ trimethylbenzoyl) phosphine oxide, was added to the preceramic 
polymers to make them photosensitive. After stereolithography, the parts were pyrolyzed at $1100{ }^{\circ} \mathrm{C}$ in nitrogen at $40{ }^{\circ} \mathrm{C} \mathrm{h}^{-1}$. The authors observed a nearly fully dense product free of macroscopic voids and defects after pyrolysis.

In this work, three preceramic formulations were introduced for potential implementation in AM. The photocuribility were investigated initially using a high power UV lamp to have a better understanding of polymer-UV interaction. Later, the polymeric formulations were tested for the actual 3D process. The rheological properties of the polymeric precursors, including the viscosity within a wide shear rate range, were compared to determine the suitability for AM technologies. The structural properties of the polymeric precursors and their photocureability were investigated using Fourier transform infrared spectroscopy, differential scanning calorimetry, and transmission electron microscopy. Moreover, thermogravimetric analysis was utilized to study the polymeric to the ceramic conversion of these three versatile precursors.

\section{Experimental procedure}

\subsection{Materials}

Three preceramic polymer systems were examined for potential $3 \mathrm{D}$ printing of ceramic parts. One is a polycarbosilane, commercially available as StarPCS ${ }^{\mathrm{TM}}$ SMP-10 (Starfire Systems, USA). The other is a proprietary polydimethylsiloxane (PDMS) UV curing formulation, commercially available as KER 4690A/B (Shin-Etsu Chemical Company, Japan). The third polymeric system is a mixture prepared from SMP-10 and a multifunctional acrylate monomer, 1,6-hexanediol diacrylate (HDDA) supplied by Sigma-Aldrich (technical grade). This reactive diluent serves two functions - reducing viscosity and accelerating the cure cycle since the unsaturated side groups make these polymers excellent candidates for free radical addition type cures. While KER $4690 \mathrm{~A} / \mathrm{B}$ is a commercially available UV curable polymer, SMP-10 and SMP-10/HDDA require the addition of a curing agent to make them UV-curable. To do so, a commercially available photoinitiator, phenylbis $(2,4,6-$ trimethylbenzoyl) phosphine oxide (a bisacylphosphine oxide, BAPO) supplied by Sigma-Aldrich, was used for SMP-10 and SMP-10/HDDA photopolymerization. After adding $2 \mathrm{wt} \%$ to the photoinitiator, the mixture was contained in amber vials and used immediately after thorough mixing to minimize exposure to UV light. The chemical structures of all components used in the three preceramic polymer systems for this study are shown in Fig. 1.

\subsection{Processing}

Thin polymeric films were manually deposited onto glass slides to simulate $3 \mathrm{D}$ printing process which allowed for quicker evaluation of the multiple formulations and process conditions. The film thickness was controlled by using a reference adhesive tape. The three preceramic polymer formulations involve SMP-10, SMP-10/HDDA (1 wt\%:1 wt\%), and PDMS ( $1 \mathrm{wt} \%$ KER 4690A, $1 \mathrm{wt} \%$ KER 4690B). After mixing for one hour, the formulations were spread onto a glass slide to produce a thin film which was followed by UV illumination for 10 seconds or 30 seconds, depending on the formulation. The cured film was subsequently peeled from the glass substrate, thermally and structurally characterized, and then transferred to the tube furnace for ceramitization under inert conditions. A tube furnace (Thermo Scientific, Linderg Blue $\mathrm{M}$, USA) with $1500{ }^{\circ} \mathrm{C}$ maximum temperature was utilized to pyrolyze the cured samples. The pyrolysis cycle involved heating from room temperature to $1350{ }^{\circ} \mathrm{C}$ under argon gas with a heating rate of $10{ }^{\circ} \mathrm{C} \mathrm{min}{ }^{-1}$. Fig. 2 illustrates the experimental workflow used to evaluate the suitability of the three preceramic polymer systems for use in AM.

To confirm the printability of the preceramic formulations, the DLP additive manufacturing technique using ANYCUBIC Photon $\mathrm{S}$, Shenzhen, with a UV-LED light source (50 W, $\lambda=405 \mathrm{~nm}$ ) was employed to $3 \mathrm{D}$ print parts with different geometries.
SMP-10

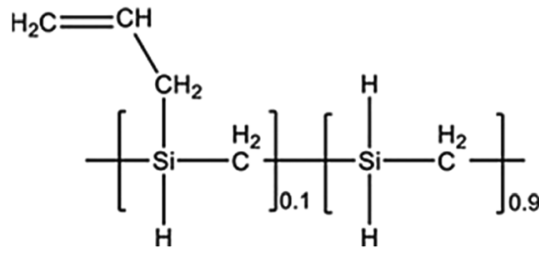

HDDA

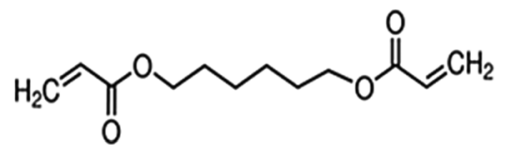

PDMS<smiles>C[Si](C)(C)O[Si](C)(C)O[Si](C)(C)C</smiles>

BAPO<smiles>Cc1cc(C)c(C(=O)P(=O)(C(=O)c2c(C)cc(C)cc2C)c2ccccc2)c(C)c1</smiles>

Fig. 1 Chemical structures of all components used in the three preceramic polymer systems. The formulation of KER 4690A/B is proprietary. 


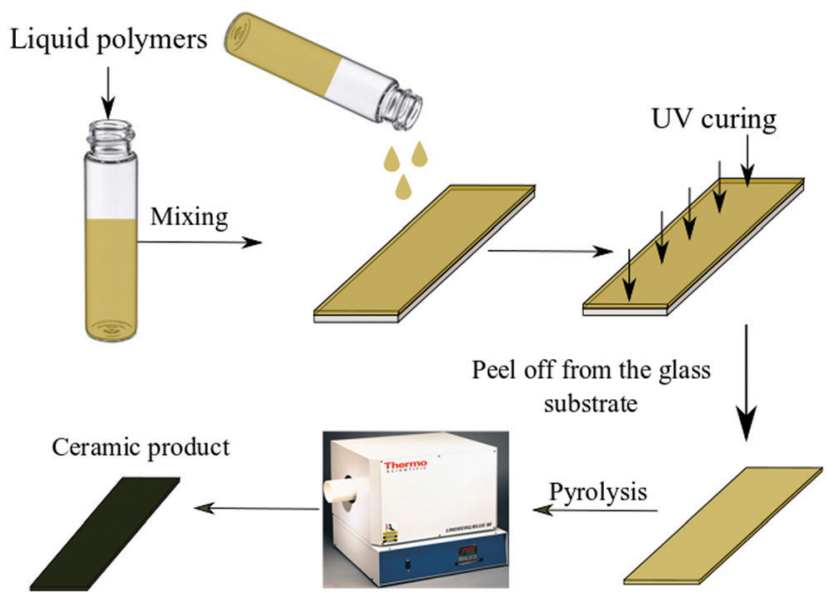

Fig. 2 Thin film deposition process - polymeric formulation deposition on a glass slide; UV exposure, and polymer to ceramic conversion in a tube furnace.

\subsection{Examinations}

The viscosity-shear rate relationship of the three preceramic polymers was measured using a rotational rheometer (MCR 302, Anton Paar, Graz, Austria) equipped with a $50 \mathrm{~mm}$ diameter coneplate measuring system with a temperature of $23{ }^{\circ} \mathrm{C}$ and a gap of $0.98 \mathrm{~mm}$. A UV Lamp (PortaRay Light Curing System, $400 \mathrm{~W}$, Uvitron International) with $405 \mathrm{~nm}$ wavelength was used to photo cure the preceramic polymer systems. The height between the UV lamp and glass slide was approximately 2.5 inches which result in $2300 \mathrm{~mW} \mathrm{~cm}^{-2} \mathrm{UV}$ light intensity on the sample. The effectiveness of UV curing was investigated by Differential scanning calorimetry (DSC), (Q2000, TA Instruments, New Castle, DE) with a heating rate of $10{ }^{\circ} \mathrm{C} \min ^{-1}$ from $25{ }^{\circ} \mathrm{C}$ to $400{ }^{\circ} \mathrm{C}$. Fourier transform infrared spectroscopy (FTIR) in attenuated total reflection mode was used to observe the structural changes that resulted from UV cure. The FTIR spectrometer (Nicolet 50-FTIR, Thermo Scientific, Waltham, MA) was set to scan 64 times with a resolution of $4 \mathrm{~cm}^{-1}$ in the range of $500-4000 \mathrm{~cm}^{-1}$. The ceramic yield of the precursors was measured using thermogravimetric analysis (TGA) in a nitrogen atmosphere. The thermogravimetric analyzer (Q500, TA Instruments, New Castle, DE) was programmed to ramp from room temperature to $900{ }^{\circ} \mathrm{C}$ at $10{ }^{\circ} \mathrm{C} \mathrm{min}{ }^{-1}$. $\mathrm{X}$-ray diffraction (XRD) measurements were used to track phase formation and was carried out using a diffractometer (Rigaku SmartLab ${ }^{(\mathbb{R}}$ ) with a voltage of $40 \mathrm{kV}$ and a current of $44 \mathrm{~mA}$. Data was collected using CuK $\alpha 1$ radiation $(\lambda=1.541 \AA$ ), a 0.04 step size, and a $2 \theta$ scan range from $5^{\circ}$ to $90^{\circ}$. The microstructural features were examined using high-resolution scanning electron microscopy (HR-SEM,) and transmission electron microscopy (TEM; Talos F200X, FEI)

\section{Results and discussion}

\subsection{Viscosity test}

Since the resin viscosity plays a vital role in determining the suitability for AM technology, the viscosities of the three preceramic polymer systems, namely SMP-10, SMP-10/HDDA, and PDMS, were investigated at a shear rate from 1 to $1000 \mathrm{~s}^{-1}$.
For stereolithography (SLA) and digital light processing (DLP) 3D printing technologies where each layer builds onto the previously cured layer, the polymer should display a low viscosity and near Newtonian behavior. ${ }^{13}$ The photosensitive resins must be able to quickly flow to the print area and selflevel to be effective using these technologies.

Overall, It can be seen from Fig. 3 that all the employed polymer systems displayed Newtonian behavior, and the resulting viscosities of SMP-10 and SMP-10/HDDA were much lower than PDMS which makes them more ideal for SLA and DLP AM technologies. Such technologies are ideal for polymers with low viscosity, typically below 3-5 Pa $\mathrm{s}^{13-15}$ The restively high viscosity of PDMS would hinder the resin flow, increase the printing time, and negatively influence the recoating efficiency. Thus, it was determined that PDMS is not a potential resin for SLA, DLP, or any vat-based AM. PDMS might be a better candidate for direct ink writing technologies that require viscous resins (range between 0.1 to $10^{3} \mathrm{~Pa} \mathrm{~s}$ ). ${ }^{5,16}$

\subsection{Crosslinking of preceramic polymers}

Being a low vinyl-containing preceramic polymer, the polymerization rate and photo-curing depth of SMP-10 are limited. Thus, the polymerization requires a high energy density UV illumination. ${ }^{14}$ Furthermore, unlike PDMS and SMP-10/HDDA, SMP-10 is translucent amber which has a slight contribution in hindering UV light penetration through the material thickness (Fig. 4a). In comparison, SMP-10/HDDA is more transparent than SMP-10 while PDMS is transparent colorless, making it even more responsive to UV illumination.

Exposing PDMS and SMP-10/HDDA polymer systems to UV light for 10 seconds, a tack-free film of $50 \mu \mathrm{m}$ thickness was formed; however, SMP-10 required more than 30 seconds of UV exposure to partially cure. This is confirmed by DSC analysis, Fig. 5, as PDMS and SMP-10/HDDA showed flat heat flow after curing. However, after 30 seconds of exposure to UV

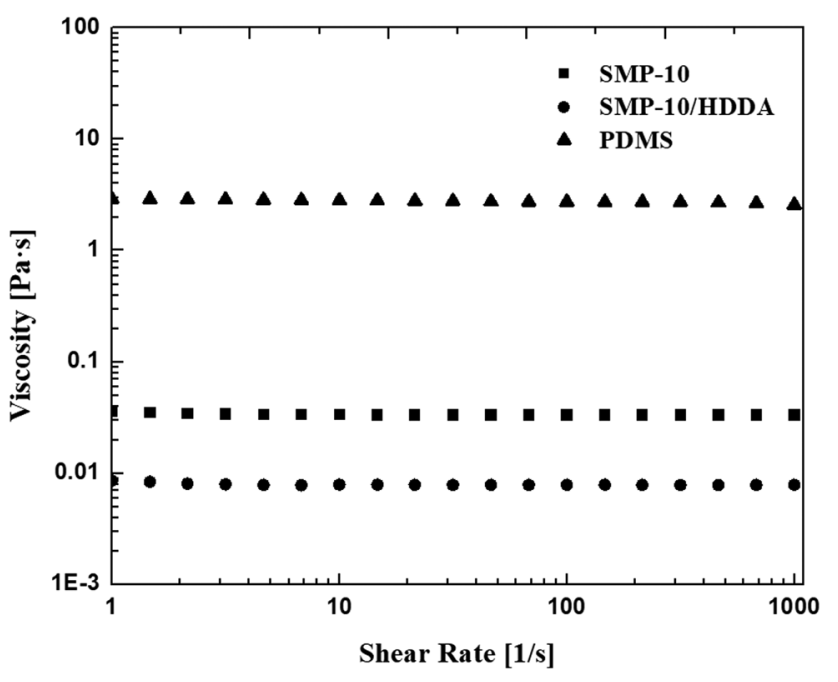

Fig. 3 Viscosity-shear rate relationship of the three preceramic polymer systems. 

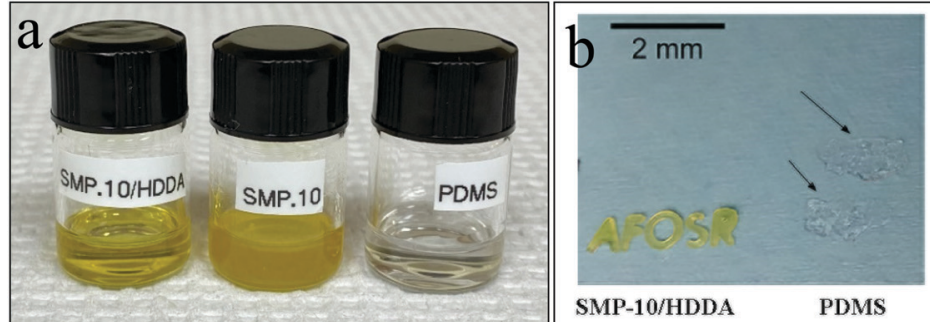

SMP-10/HDDA

PDMS

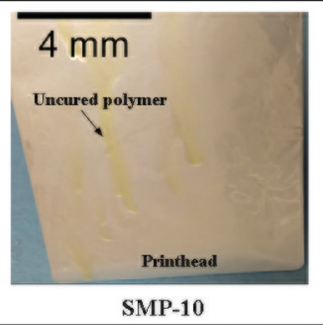

Fig. 4 The appearance of polymeric formulations (a) after mixing (b) after 3D printing - successful printing of SMP-10/HDDA, incomplete curing with printing low-resolution of PDMS, and lack of SMP-10 reaction with UV light.

illumination, SMP-10 showed an exothermic peak at approximately $230{ }^{\circ} \mathrm{C}$, indicating the SMP-10 was only partially cured.

Using a 3D printer with lower UV intensity to print the polymeric formulations requires increasing the UV exposure time to control the total UV dose delivered to the material. Printing SMP.10/HDDA proved excellent UV-response and highresolution printing (Fig. 4b) with a minute exposure time. However, SMP-10 showed no curing even at a longer exposure time up to $3 \mathrm{~min}$. Even though PDMS exhibits incomplete curing at a shorter curing time, it showed full curing after three minutes of illumination with poor resolution printing due to the high viscosity of PDMS (Fig. 4b).

FTIR results of SMP-10 before and after exposure to UV light (Fig. 6a) show that the peak around $1630 \mathrm{~cm}^{-1}$, which is attributed to the $\mathrm{C}=\mathrm{C}$ stretch of the silicon-allyl group

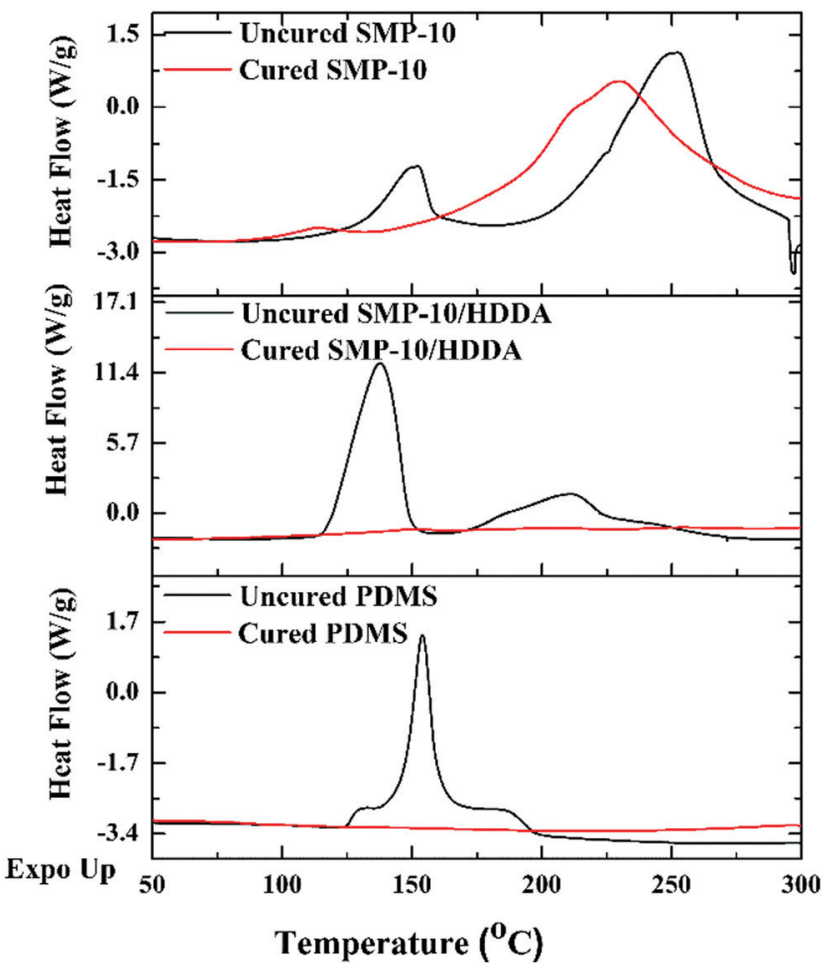

Fig. 5 DSC curves before and after UV exposure for the three formulations illustrating the disappearance of exothermic heat flow peak of SMP10/HDDA and PDMS after UV exposure and slight changes in SMP-10 peak intensity after UV exposure due to it is poor interaction with UV light.
(Si-allyl) ${ }^{12}$ was reduced upon UV exposure. The formation of the carbonyl $(\mathrm{C}=\mathrm{O})$ peak after curing SMP-10 may be attributed to either the carbonyl of the photoinitiator and/or oxidation of the silicon-hydrogen ( $\mathrm{Si}-\mathrm{H})$ or silicon-methylene $\left(\mathrm{Si}^{-} \mathrm{CH}_{2}-\right)$ groups, considered active sites for vinyl photopolymerization. ${ }^{14}$ The addition of HDDA to SMP-10 contributed to an increase in the number of vinyl $(\mathrm{C}=\mathrm{C})$ groups (Fig. $6 \mathrm{~b})$ in comparison to pure SMP-10 due to the acrylate of HDDA. As seen in Fig. 6b, the $\mathrm{C}=\mathrm{C}$ bonds of the acrylate group, appearing as a doublet peak (1635 $\mathrm{cm}^{-1}$ and $1619 \mathrm{~cm}^{-1}$ ), effectively disappeared after UV illumination for $10 \mathrm{sec}$ which indicates the HDDA fully reacted and a successful co-photopolymerization took place. According to the manufacturer, KER 4690AB is cured by hydrosilylation under a photoactivated Pt catalyst, which arises between $\mathrm{Si}-\mathrm{H}$ and vinyl groups within the resin (Fig. 6c).

\subsection{Polymer to ceramic conversion}

The polymer to the ceramic conversion of the successfully printed and pyrolyzed parts to $1350{ }^{\circ} \mathrm{C}$, SMP-10/HDDA, showed uniform shrinkage without noticeable macroscopic defects. An estimated linear shrinkage of around $30 \%$ was recorded for such a sample (Fig. 7).

To track the major events during the polymer to the ceramic conversion of cross-linked PDMS, SMP-10, and SMP-10/HDDA, TGA, and XRD were employed. From TGA analysis (Fig. 8), SMP-10 showed the lowest onset temperature and the highest ceramic yield. The decomposition of SMP-10 appeared to occur at three temperatures: $157{ }^{\circ} \mathrm{C}, 440{ }^{\circ} \mathrm{C}$, and $749{ }^{\circ} \mathrm{C}$. An initial weight loss of approximately $9 \mathrm{wt} \%$ is attributed to incomplete hydrosilylation and dehydrocoupling from UV crosslinking, which occurs at the first stage of pyrolysis. Thus, the evaporation of hydrogen gas and short oligomers are the major events at this temperature. Massive weight loss of around $20 \mathrm{wt} \%$ is associated with the second stage of pyrolysis due to the evolution of both hydrogen and methane gases. ${ }^{12}$ In the third stage, there was a slight weight loss due to $\mathrm{Si}-\mathrm{C}$ and $\mathrm{Si}-\mathrm{O}-\mathrm{C}$ bridging formations, which means that the polymer to ceramic conversion occurred beyond this temperature. ${ }^{17}$

A dramatic weight loss (around $30 \mathrm{wt} \%$ ) between 400 and $500{ }^{\circ} \mathrm{C}$ is connected with the HDDA addition to SMP-10. The mass loss is attributed to small moieties separation from the acrylate and preceramic polymer main chain at this temperature range. 

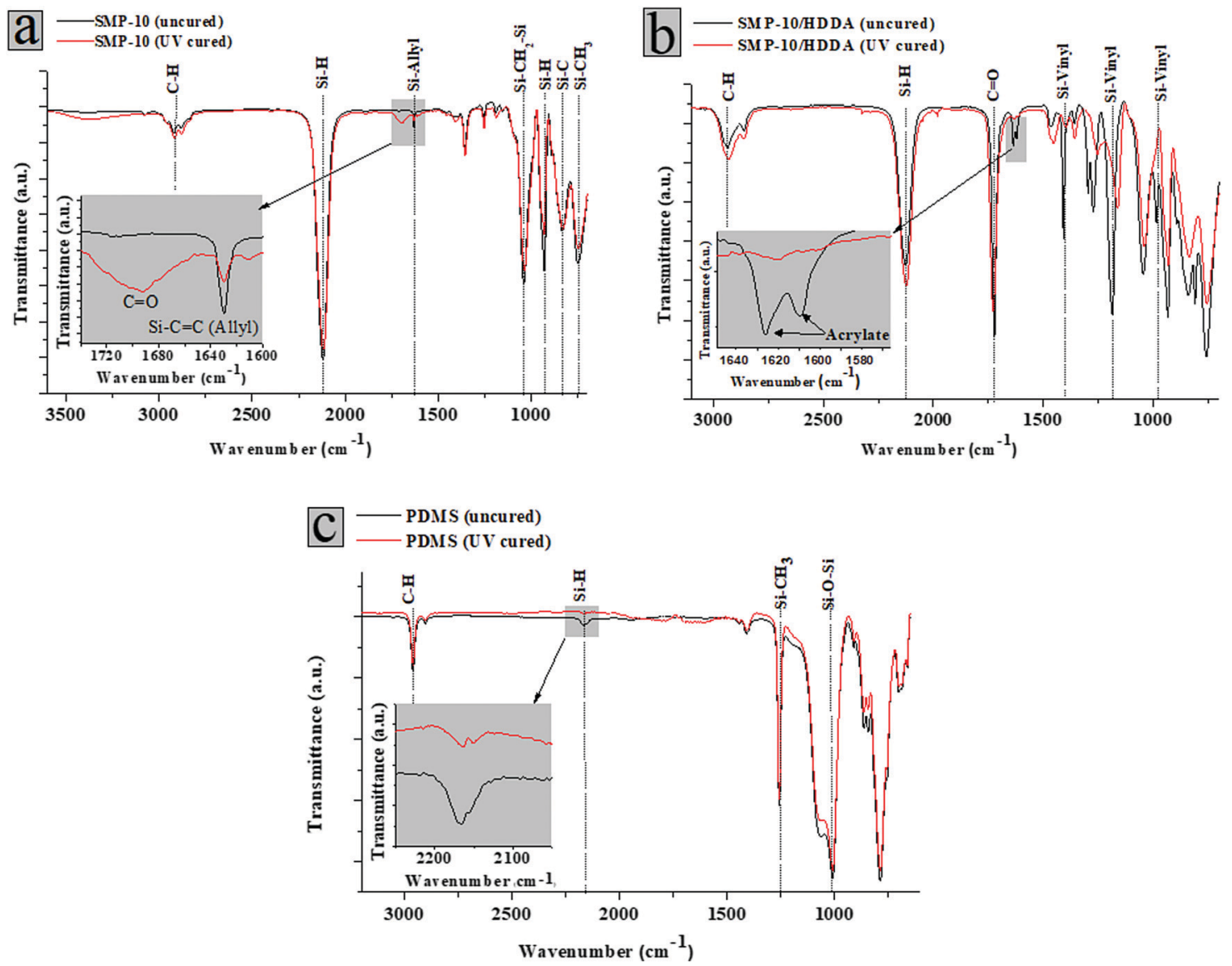

Fig. 6 FTIR spectroscopy results for (a) SMP-10, (b) SMP-10/HDDA, (c) PDMS.

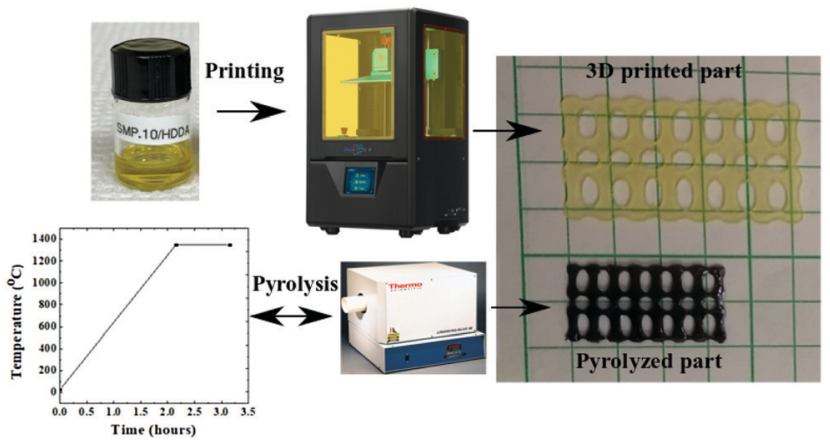

Fig. 7 Outline of 3D printing/pyrolysis process of SMP-10/HDDA showing the ability to print complex geometries from such formulation and ability to survive the pyrolysis cycle.

On the other hand, PDMS showed the highest onset temperature and lowest ceramic yield with major depolymerization temperature between $400-750{ }^{\circ} \mathrm{C}$. During this range, most PDMS converted to cyclic oligomers through two mechanisms. At lower temperature ranges around $400-500{ }^{\circ} \mathrm{C}$, unzip degradation is the dominant mechanism generating cyclic siloxanes. At temperatures over $500{ }^{\circ} \mathrm{C}$, rearrangement degradation proceeds by readjustment of $\mathrm{Si}-\mathrm{O}-\mathrm{Si}$ bonds in the siloxane backbone and heterolytic cleavage. The subsequent species from this degradation are cyclic siloxanes and short moieties. ${ }^{18}$

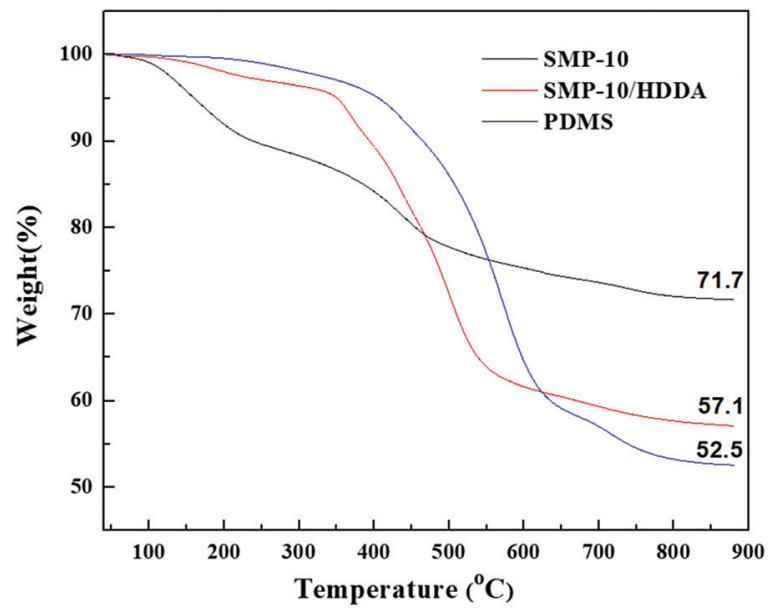

Fig. 8 TGA curves for different preceramic polymers formulations.

Based on the TGA results (acceptable weight retention of SMP-10/HDDA) along with aforementioned features such as excellent viscosity and curability showed this formulation to be the best candidate for SLA and DLP 3D printing. Although the pure SMP-10 showed outstanding retained weight, its limited photocuribility excluded it as a candidate for our 3D printing process. 
To track the ceramic phase's formation including SiC and SiOC, XRD analysis was used. The common SiC structures produced from polycarbosilane polymers are cubic silicon carbide ( $\beta$-SiC) and hexagonal silicon carbides $(\alpha$-SiC). At temperatures below $1700{ }^{\circ} \mathrm{C}, \beta$-SiC is the dominant phase, while $\alpha$-SiC begins to form at higher temperatures. Since the current study investigates the phase formations at temperatures as low as $1350{ }^{\circ} \mathrm{C}$, the expected $\mathrm{SiC}$ from XRD is $\beta$-SiC. From Fig. 9, SMP-10, and SMP-10/HDDA displayed characteristic peaks at $36.5^{\circ}(111), 60.1^{\circ}(220)$, and $73^{\circ}$ (311) which is recognized as $\beta$-SiC with a zinc blende crystal structure. ${ }^{19}$ The addition of HDDA to SMP-10 results in the presence of a halo at $16-25^{\circ}$ due to the $-\mathrm{Si}-\mathrm{O}-/-\mathrm{Si}-\mathrm{O}-\mathrm{C}$ formation. The internal oxygen in the acrylate (HDDA) structure is the main source of SiOC formation. On the other hand, PDMS showed a less characteristic SiC XRD due to the Si-O linkage in siloxane which hinders SiC formation. In conclusion, the SMP-10/ HDDA polymeric formulation showed an excellent structural and physical combination which was proved by a well-defined SiC phase formation, sensitivity to UV illumination, and low viscosity.

While XRD provided qualitative information about the crystallinity of the bulk samples, HR-TEM characterization gives a representation regarding crucial structural features such as lattice fringes, diffraction patterns, and elemental mapping. Such characteristics for all formulations in this study were investigated by utilizing HR-TEM equipped with EDS in STEM mode. All pyrolyzed formulations showed the existence of $\mathrm{SiC}$ and SiOC phases as shown in Fig. 10. A highly ordered lattice on (111) SiC plane was observed from TEM lattice fringe imaging which was confirmed by calculating the interatomic spacing of $0.25 \mathrm{~nm}$. Noticeable differences in the SiC phase size and distribution were observed for the different formulations. SMP-10/HDDA proved more extended SiC phase ordering as compared with SMP-10 and PDMS.

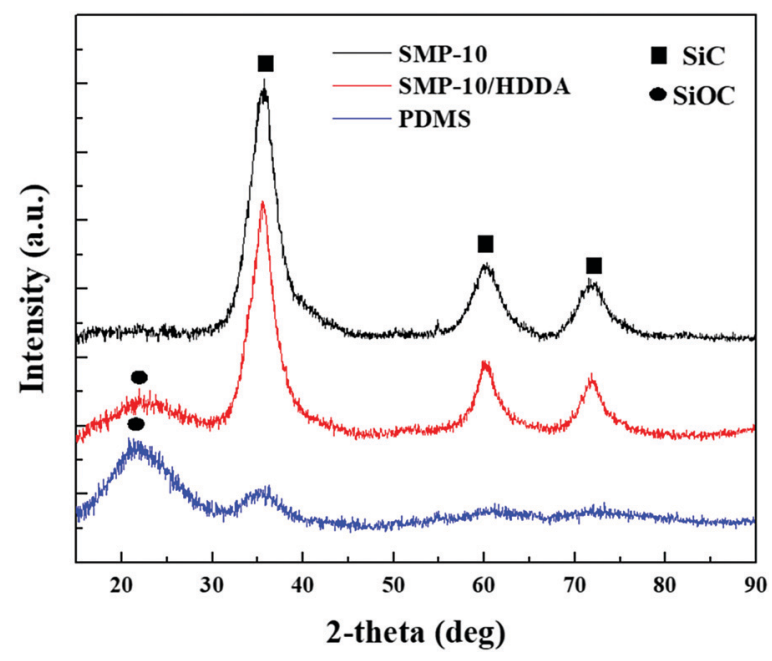

Fig. 9 XRD curves for different preceramic polymers formulations.
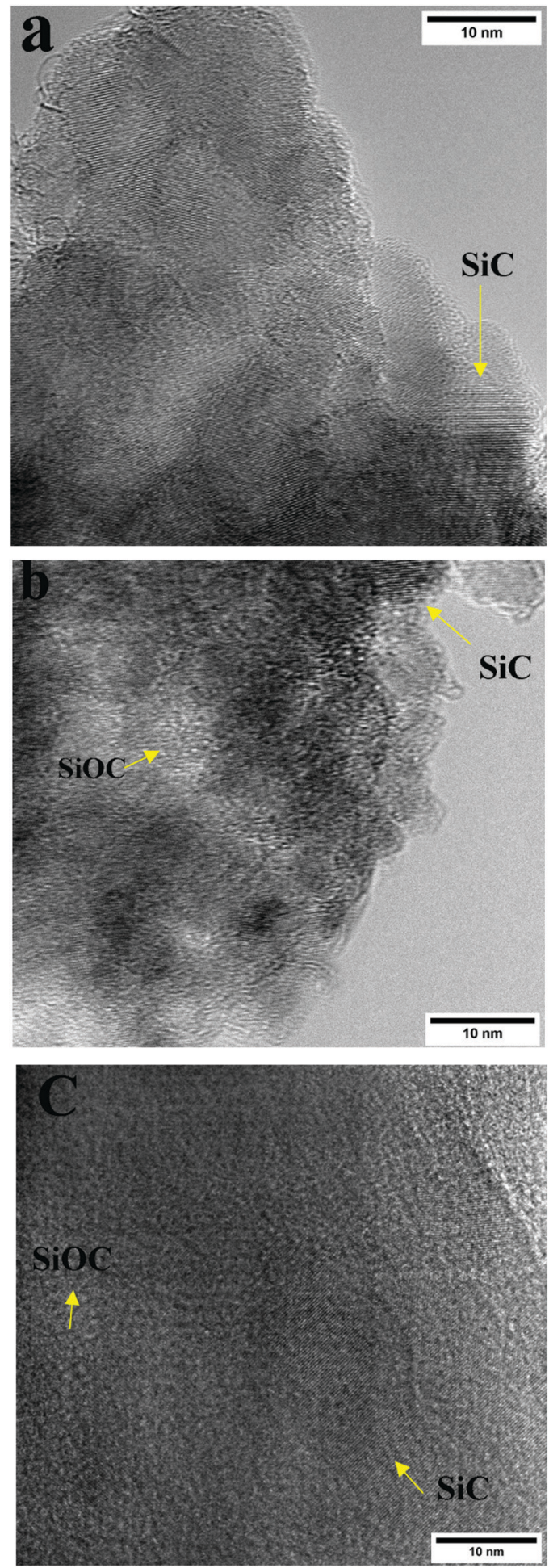

Fig. 10 (a) HR-TEM lattice fringes imaging for (a) SMP-10/HDDA, (b) SMP-10, (c) PDMS, images prepared using the powdered sample on lacey carbon TEM grid.

\section{Conclusions}

The feasibility of additive manufacturing of three polymerderived ceramic formulations, namely SMP-10, SMP-10/HDDA, and PDMS, was investigated in this study. To do so, structural 
and physical examinations of the polymeric precursors were investigated. Viscosity tests for different formulations were compared to confirm the suitability for specific additive manufacturing techniques, with SMP-10/HDDA showing the most suitable viscosity. The same formulation demonstrated outstanding photocurbility as shown by FTIR and DSC analyses. Thermal gravimetric analysis (TGA) results showed an outstanding retained weight of slightly more than 70\% for SMP-10 and a retained weight of nearly $60 \%$ for SMP-10/HDDA. SMP-10 and SMP-10/HDDA showed characteristic SiC peaks from XRD analysis and well-ordered SiC interatomic planes from TEM lattice fringe imaging, as compared with the PDMS material. The Si-O linkage in PDMS hinders SiC formation. Furthermore, SMP-10/HDDA showed low viscosity (around 0.01 Pa s) and outstanding photocurability, which makes it an excellent candidate for polymer-based AM technologies.

\section{Conflicts of interest}

There are no conflicts to declare.

\section{Acknowledgements}

This effort is funded by the Air Force Office of Scientific Research (AFOSR) Grant FA9550-18-1-0274, thanks to Drs. Goretta and Pan for their support of this program. Thanks to the Electron Microscopy Center, the University of Kentucky, which belongs to the National Science Foundation NNCI Kentucky Multiscale Manufacturing and Nano Integration Node, supported by ECCS-1542174, for their collaboration to facilitate TEM characterization.

\section{References}

1 S. Shahrestani, M. C. Ismail, S. Kakooei and M. Beheshti, Effect of additives on slip casting rheology, microstructure and mechanical properties of $\mathrm{Si}_{3} \mathrm{~N}_{4} / \mathrm{SiC}$ composites, Ceram. Int., 2020, 46, 6182-6190.

2 C. W. Gal, G. W. Song, W. H. Baek, H. K. Kim, D. K. Lee, K. W. Lim and S. J. Park, Fabrication of pressureless sintered Si3N4 ceramic balls by powder injection molding, Ceram. Int., 2019, 45, 6418-6424.

3 M. S. Asl, B. Nayebi, M. G. Kakroudi and M. Shokouhimehr, Investigation of hot pressed ZrB2-SiC-carbon black nanocomposite by scanning and transmission electron microscopy, Ceram. Int., 2019, 45, 16759-16764.

4 L. Feng, W. G. Fahrenholtz and G. E. Hilmas, Effect of ZrB2 content on the densification, microstructure, and mechanical properties of ZrC-SiC ceramics, J. Eur. Ceram. Soc., 2020, 40, 220-225.

5 L. Li, Q. Lin, M. Tang, A. J. E. Duncan and C. Ke, Advanced Polymer Designs for Direct-Ink-Write 3D Printing, Chem. Eur. J, 2019, 25, 10768-10781, DOI: 10.1002/chem.20190 0975 .
6 P. J. Bártolo, Stereolithography: materials, processes and applications, Springer Science \& Business Media, 2011, ISBN 0387929045.

7 G. Franchin, H. S. Maden, L. Wahl, A. Baliello, M. Pasetto and P. Colombo, Optimization and Characterization of Preceramic Inks for Direct Ink Writing of Ceramic Matrix Composite Structures, Mater, 2018, 11, 515, DOI: 10.3390/ ma11040515.

8 E. Kroke, Y.-L. Li, C. Konetschny, E. Lecomte, C. Fasel and R. Riedel, Silazane derived ceramics and related materials, Mater. Sci. Eng., R, 2000, 26, 97-199, DOI: 10.1016/S0927796X(00)00008-5.

9 M. Birot, J.-P. Pillot and J. Dunogues, Comprehensive Chemistry of Polycarbosilanes, Polysilazanes, and Polycarbosilazanes as Precursors of Ceramics, Chem. Rev., 1995, 95, 1443-1477, DOI: 10.1021/cr00037a014.

10 Z. C. Eckel, C. Zhou, J. H. Martin, A. J. Jacobsen, W. B. Carter and T. A. Schaedler, Additive manufacturing of polymerderived ceramics, Science, 2016, 351, 58-62, DOI: 10.1126/ science.aad2688.

11 M. Wang, C. Xie, R. He, G. Ding, K. Zhang, G. Wang and D. Fang, Polymer-derived silicon nitride ceramics by digital light processing based additive manufacturing, J. Am. Ceram. Soc., 2019, 102, 5117-5126, DOI: 10.1111/jace.16389.

12 X. Wang, F. Schmidt, D. Hanaor, P. H. Kamm, S. Li and A. Gurlo, Additive manufacturing of ceramics from preceramic polymers: A versatile stereolithographic approach assisted by thiol-ene click chemistry, Addit. Manuf., 2019, 27, 80-90, DOI: 10.1016/j.addma.2019.02.012.

13 J. W. Halloran; M. Griffith and T.-M. Chu, Stereolithography resin for rapid prototyping of ceramics and metals, 2000.

$14 \mathrm{X}$. Wang, Photoinduced thiol-ene click chemistry assisted additive manufacturing and freeze casting of polymer-derived ceramics, Universitätsverlag der TU Berlin, 2019, vol. 3, ISBN 3798330883.

15 G. Taormina, C. Sciancalepore, M. Messori and F. Bondioli, $3 \mathrm{D}$ printing processes for photocurable polymeric materials: technologies, materials, and future trends, J. Appl. Biomater. Funct. Mater., 2018, 16, 151-160, DOI: 10.1177/ 2280800018764770.

16 K. Hölzl, S. Lin, L. Tytgat, S. Van Vlierberghe, L. Gu and A. Ovsianikov, Bioink properties before, during and after 3D bioprinting, Biofabrication, 2016, 8, 32002, DOI: 10.1088/ 1758-5090/8/3/032002.

17 G. D. Soraru, F. Babonneau and J. D. Mackenzie, Structural evolutions from polycarbosilane to SiC ceramic, J. Mater. Sci., 1990, 25, 3886-3893, DOI: 10.1007/BF00582455.

18 G. Camino, S. M. Lomakin and M. Lazzari, Polydimethylsiloxane thermal degradation Part 1. Kinetic aspects, Polymer, 2001, 42, 2395-2402, DOI: 10.1016/S0032-3861(00)00652-2.

19 T.-E. Kim, K.-E. Khishigbayar and K. Y. Cho, Effect of heating rate on the properties of silicon carbide fiber with chemical-vapor-cured polycarbosilane fiber, J. Adv. Ceram., 2017, 6, 59-66, DOI: 10.1007/s40145-017-0218-4. 